\title{
Effect of Pillar and Mesh Structure of Tetrahedral Amorphous Carbon (ta-C) Coatings on the Wear Properties and Fracture Toughness of the Coating
}

\author{
Mohd Muhyiddin Bin Mustafa ${ }^{1,3)^{*}}$, Noritsugu Umehara ${ }^{1)}$, Takayuki Tokoroyama ${ }^{1)}$, Motoyuki Murashima ${ }^{1)}$, \\ Akinori Shibata ${ }^{2)}$, Yoshiharu Utsumi ${ }^{2)}$ and Hideki Moriguchi ${ }^{2)}$ \\ ${ }^{1)}$ Department of Micro-Nano Mechanical Science and Engineering, Graduate School of Engineering, \\ Nagoya University, Furo-cho, Chikusa-ku, Nagoya, Aichi 464-8603, Japan \\ ${ }^{2)}$ Technology Development Center, Nippon ITF Inc., 47, Umezu-Takase-cho, Ukyo-ku, Kyoto 615-8686, Japan \\ ${ }^{3)}$ Faculty of Mechanical and Manufacturing Engineering Technology, Universiti Teknikal Malaysia Melaka, \\ Hang Tuah Jaya, 76100 Durian Tunggal, Melaka, Malaysia \\ *Corresponding author: Mohd Muhyiddin Bin Mustafa (muhyiddin@ume.mech.nagoya-u.ac.jp)
}

Manuscript received 05 July 2019; accepted 28 August 2019; published 15 December 2019

Presented at the International Tribology Conference Sendai 2019, 17-21 September, 2019

\begin{abstract}
Wear by fracture is among the factors associated with the DLC coating failures in the tribological application. The current study investigated the link between the wear and the fracture-toughness on the novel Pillar and Mesh structure ta- $\mathrm{C}$ coatings, in addition to conventional ta-C coatings. The tribological properties of these coatings were examined under base-oil lubrication via ball-on-disk tribo-tester and micro indentation technique was used to characterize the fracture toughness. The wear track and the indentation mark were analyzed using the optical microscope, 3D laser microscope and FE-SEM. The friction coefficient for ta-C, Pillar ta-C and Mesh ta-C are within the range of 0.071 to 0.106 . Mesh ta-C indicated the highest wear resistance, followed by the Pillar ta- $\mathrm{C}$ and conventional ta-C. Also, Mesh ta-C demonstrated the highest fracture-toughness value with $16.6 \mathrm{MPa} \cdot \mathrm{m}^{1 / 2}$, followed by Pillar ta-C with $13.4 \mathrm{MPa} \cdot \mathrm{m}^{1 / 2}$ in contrast to ta-C. Greater resistance to wear for ta- $\mathrm{C}$ with Pillar and Mesh structure was detected with an increased fracture-toughness and improvement in crack propagation inhibition. Moreover, the Pillar and Mesh ta-C provides superior rate of crack-energy dissipation as compared to the ta-C.
\end{abstract}

\section{Keywords}

diamond-like carbon coating, DLC structure, fracture toughness, wear, crack resistance, vickers indenter

\section{Introduction}

Diamond-like carbon (DLC) is well-established in enhancing the tribological performance of the mechanical parts, especially in automotive industry to decrease both friction and wear. DLC exhibits significant features including high hardness in addition to wear resistance that makes it highly promising for tribological application. The application of the DLC coatings is commonly for the high contact pressure component. Therefore, the coating crack resistance or fracture toughness often restricts the performance or service lifetime. In general, fracture toughness refers to the capability of the material to resist the pre-existing crack growth. Toughness is the measure of energy utilised to produce crack and facilitating crack to propagate causing fracture. Meanwhile, fracture toughness is known as the energy that is needed for the crack propagation to failure [1]. Frequently, toughness is known as the energy absorption capacity of a film material for the duration of the transition from deformation to fracture. Toughness is an essential mechanical property with regard to the cohesive force of DLC film in which it indicates the film capacity to resist the formation of cracks near the defects in the film caused by the stress accumulation [2].

The mechanical properties are well-known to limit the performance and reliability of a thin coating like DLC. Application of DLC coating such in the automotive components by means of rubbing of two components leads to enhancing the features including the friction and wear characteristics. Therefore, there have been increased studies on enhancing the tribological characteristics of the coatings through modifying the deposition methods and doping element. Nevertheless, there are several limitations in evaluating of the DLC coating wear performance via the fracture toughness. Evidence has shown that harder coatings like ta-C are highly brittle and can be readily fractured by high-pressure contact throughout the application. Hence, in order to examine the brittleness of the thin coating, it is crucial to quantify the fracture toughness. 

of the Coating

Hardness is among the key factors in regulating the wear performance of the DLC coating. As such, high hardness with low crack resistance is associated with severe wear due to through-thickness crack [3]. Similarly, high $\mathrm{sp}^{2}$ carbon atoms DLC coating that is low in hardness could lead to high wear as the results of structural alteration including graphitization of the contact surface under severe condition $[4,5]$. DLC is frequently deposited with a layer of homogeneous structure excluding multilayer coating pattern. Therefore, tailoring the properties, particularly the hardness by employing the DLC structure could promote superior wear performance.

The DLC coating is associated with the deposition of the coating onto a substrate. Therefore, the technique that was employed to examine the fracture toughness for the bulk material is not feasible for evaluating the fracture toughness for the coating [6]. It should be noted that the quantification of the fracture toughness of the coating is still challenging owing to thickness limitation of the DLC coating [7]. The measurement of the crack length during indentation could be utilised to evaluate the fracture toughness. On that account, the current study applied Vickers indenter as it has been demonstrated to form bigger radial cracks that provide better accuracy of cracks length measurement. Thus, the radial crack formed follows the axis of diagonal of the indent and restraint the coating from secondary crack growth and chipping $[8,9]$. Plastic zone that is prolonged under loading condition deploys the tensile stress into the coating. Moreover, additional stress is generated throughout the unloading condition when the elastically strained coating initiated to resume to its actual shape. Nonetheless, the aforementioned process is limited by the permanent deformation linked to the plastic zone [10]. Radial crack on the coating is generated during unloading process, which forms the residual tensile stresses [11]. Frequently, indentation on specimen leads to both elastic and plastic deformations. In brittle materials, plastic deformation generally exists with pointed indenter including Vickers.

Evidence has revealed that the alteration of the structure by multilayer coating deposition has shown to decrease the impact of the adhesive wear, improve the fracture resistance and load carrying capacity, as well as elastic recovery value $[12,13]$. The current study evaluated wear performance and the fracture toughness of conventional tetrahedral amorphous carbon (ta-C), pillar structure ta- $\mathrm{C}$ (Pillar ta-C), and mesh structure ta-C (Mesh ta-C) through micro-indentation technique. The findings of this study could provide further insight into understanding on how the fracture toughness affects the tribological performance of the coatings and how the structure of a coating results in higher fracture toughness in addition to wear resistance.

\section{Experimental details}

\subsection{Specimens}

Three types of ta-C coatings were differentiated by the structure variation; ta- $\mathrm{C}$ had the uniform structure, whereby the Pillar and Mesh ta-C were generated with pillar and mesh structure, correspondingly, as depicted in Fig. 2(a), (b), and (c). All samples were provided by the Nippon ITF Inc. The deposition of the aforementioned coatings was performed on (100) single crystalline silicon (Si-wafer) substrates by physical vapour deposition (PVD) technique.

The thickness and surface roughness of the deposited coating were determined via the surface profilometer (S-3000, Mitsuyo, Japan). Moreover, the NANOPICS 1000 Elionix ENT1100a Nanoindenter was used to determine the hardness and Young modulus of the coating. Residual stress induced through the deposition process was determined by Stoney equation by calculating the radius of curvature of the Si-wafer predeposition and post-deposition procedures. Mechanical and physical characteristics of the coating samples utilised in this study are presented in Table 1.

\subsection{Characteristic of pillar and mesh ta-C details \\ 2.2.1 Concept and preparation method}

A total of three types of ta-C coatings, namely conventional ta-C, Pillar ta-C, and Mesh ta-C were prepared using M720 PVD apparatus.

Mesh and Pillar ta-C coating were deposited through modifying the method utilised to deposit conventional ta-C coating. In the deposition process of conventional ta- $\mathrm{C}$, the cooling system was employed to maintain the temperature below $150^{\circ} \mathrm{C}$ as illustrated in Fig. 1. Similarly, the heating system was applied to increase and maintain the temperature

Table 1 Characteristics of the ta-C, pillar ta-C, and mesh structure ta- $\mathrm{C}$ coatings

\begin{tabular}{lrrrr}
\hline Samples & $\begin{array}{c}\text { Film thickness } \\
\boldsymbol{d}, \boldsymbol{\mu m}\end{array}$ & $\begin{array}{c}\text { Elastic modulus } \\
\boldsymbol{E}_{\boldsymbol{f}}, \mathrm{GPa}\end{array}$ & $\begin{array}{c}\text { Hardness } \\
\boldsymbol{H}, \mathrm{GPa}\end{array}$ & $\begin{array}{c}\text { Compressive } \\
\text { residual stress } \\
\boldsymbol{\sigma}_{\boldsymbol{c}}, \mathrm{GPa}\end{array}$ \\
\hline ta-C & 1.5 & 310 & 40.1 & 1.75 \\
Pillar ta-C & 1.42 & 143 & 18.0 & 1.26 \\
Mesh ta-C & 1.25 & 184 & 20.0 & 2.15 \\
\hline
\end{tabular}
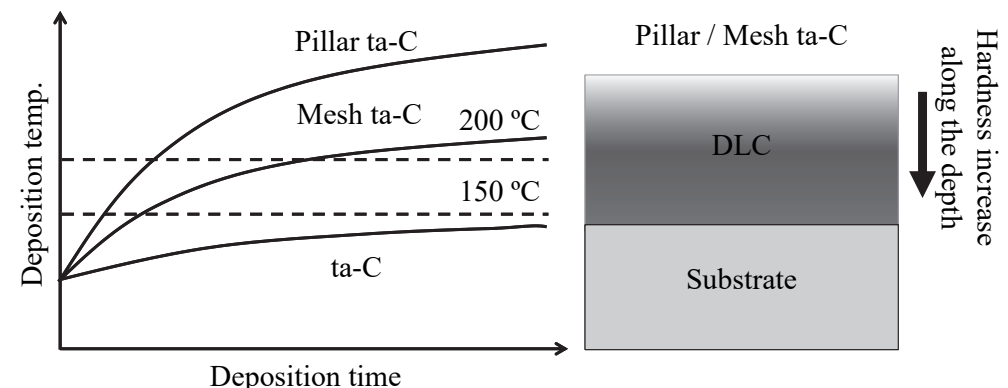

Fig. 1 Analysis of conceptual deposition technique for conventional ta-C coating, pillar and mesh ta-C DLC coating 


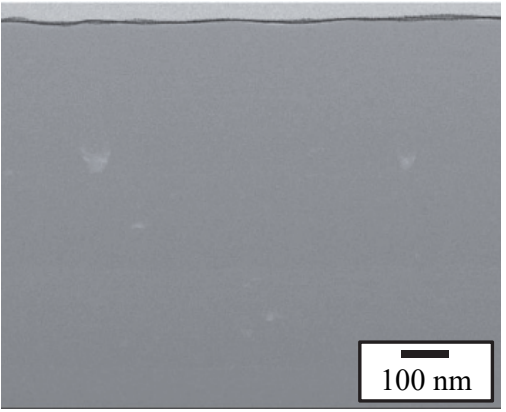

(a)

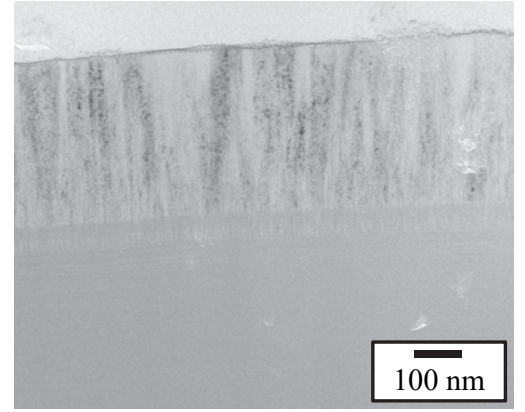

(b)

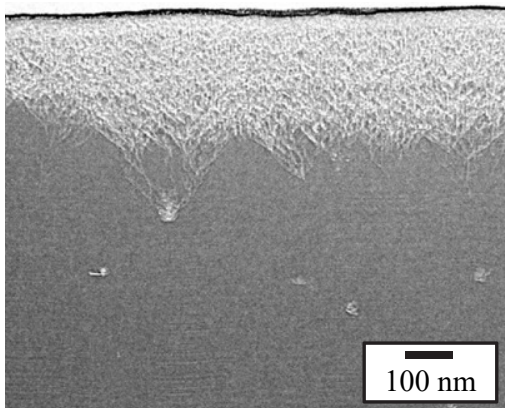

(c)

Fig. 2 Cross sectional cut of (a) conventional ta-C, (b) pillar ta-C, and (c) mesh ta-C

of the substrate at above $200^{\circ} \mathrm{C}$ throughout the process of depositing Pillar and Mesh ta-C. The application of continuous heating resulted in decline in the coating hardness when the coating thickness was increased as the graphitic structure formed during coating growth.

\subsubsection{Coating structure}

Cross-sectional image of the coating was generated through observation via the focused ion beam (FIB) under transmission electron microscope (TEM H9000 UHR), Figs. 2 and 3.

Concerning conventional ta-C cross-sectional images, Fig. 2(a) demonstrates characteristic of a homogenous structure. Moreover, the Pillar and Mesh ta-C demonstrated a reticulated structure originated from the micro particle, produced by the arc deposition method as presented in Fig. 2(b) and (c), respectively. Micro-electron diffraction analysis was performed at many spots on the cross-sectional of the Pillar ta-C and Mesh ta-C as illustrated in Fig. 4(a) and (b), correspondingly. According to the International Center for Diffraction Data (ICDD) database, crystalline graphite lattice spacing constant is $0.33756 \mathrm{~nm}$. The results of crystallinity experiment performed using electron diffraction method by TEM JEM2100F are depicted in Fig. 5(a) and (b). The Pillar ta-C and Mesh ta-C were generated nearly $0.34 \mathrm{~nm}$ and $0.35 \mathrm{~nm}$ spacing, respectively. The obtained crystallinity result is almost similar to the ICDD data which indicate the presence of crystalline graphite microstructure at the quarter of the coating thickness of Pillar ta-C and Mesh ta-C.

\section{Tribological experiments}

The friction tests were conducted using the ball-on-disk tribo-tester as presented in Fig. 6 within boundary lubrication regime with a constant normal load of $1 \mathrm{~N}$ on the SUJ-2 ball, correlated with the maximum Hertzian contact pressures of $421 \mathrm{MPa}$. The SUJ-2 ball was rubbed against coated Siwafer placed $4.0 \mathrm{~mm}$ eccentrically from the center of the disk under a pure sliding condition via fixing the SUJ-2 ball to the upper jig. Moreover, the Si-wafer was fixed on the lower holder that was located on the rotary turntable. The speed and temperature of the tests were maintained at $0.042 \mathrm{~m} / \mathrm{s}$ for 60 minutes that correlated with $150 \mathrm{~m}$ of sliding distance and $80^{\circ} \mathrm{C}$, correspondingly. Both SUJ-2 ball and counterpart Si-wafer disk were assured to submerge under the $\mathrm{PAO} 4$ oil level thereupon heat was applied to maintain the temperature of $80^{\circ} \mathrm{C}$ throughout the friction test. The friction test was performed in triplicate to guarantee validation and reproducibility of the results.

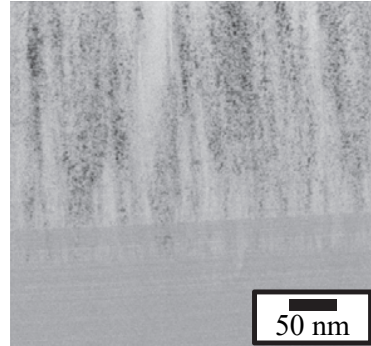

(a)

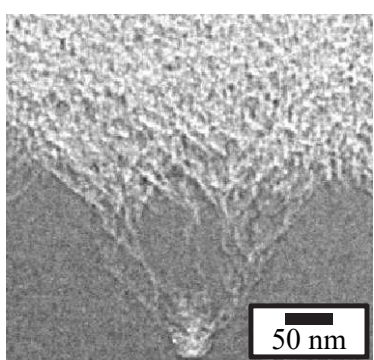

(b)
Fig. 3 Magnified images of reticulated structure for (a) pillar ta-C, and (b) mesh ta-C

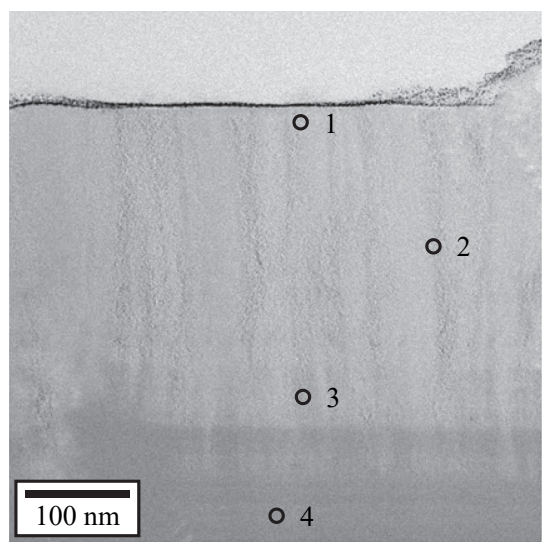

(a)

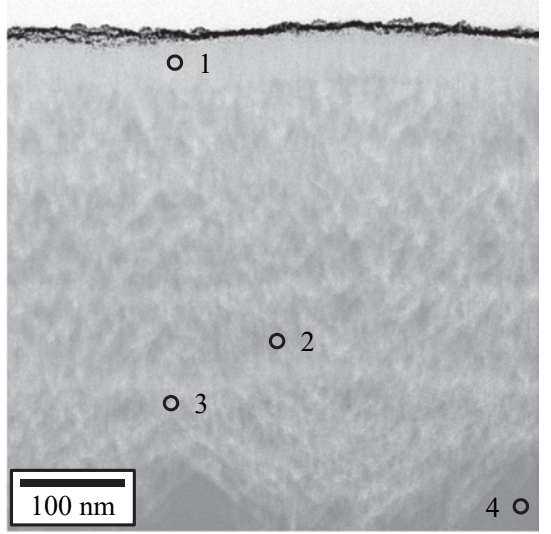

(b)

Fig. 4 Micro-electron diffraction acquisition position for (a) pillar ta-C, and (b) mesh ta-C 

of the Coating
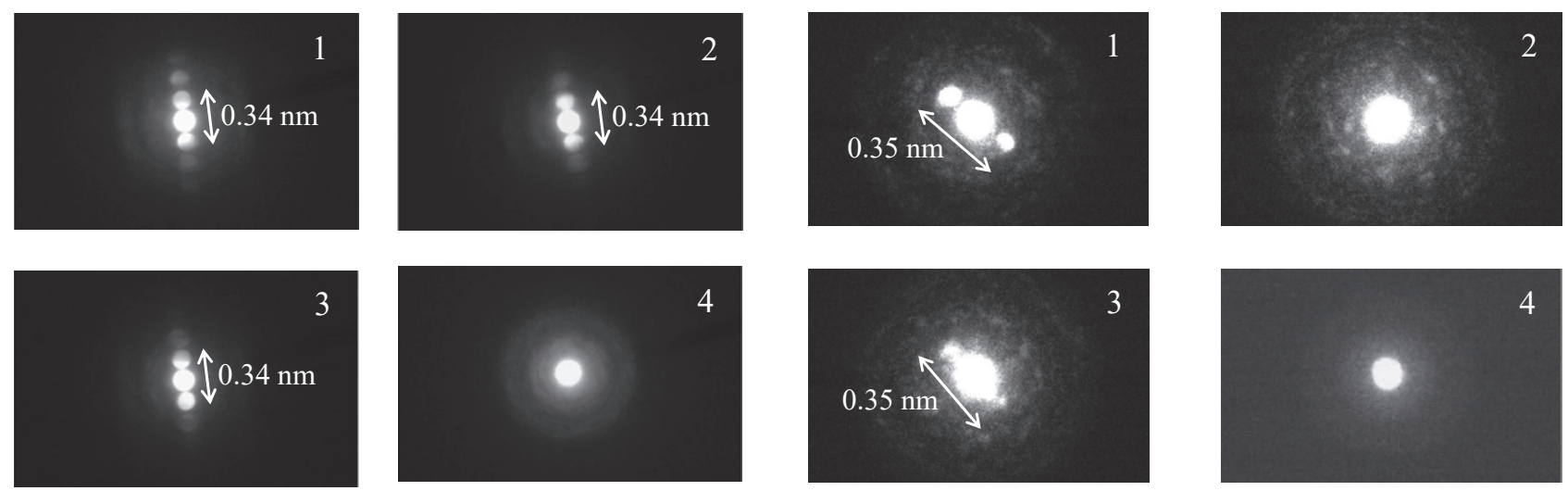

(a)

(b)

Fig. 5 Crystallinity evaluation result at different position for (a) pillar ta-C, and (b) mesh ta-C

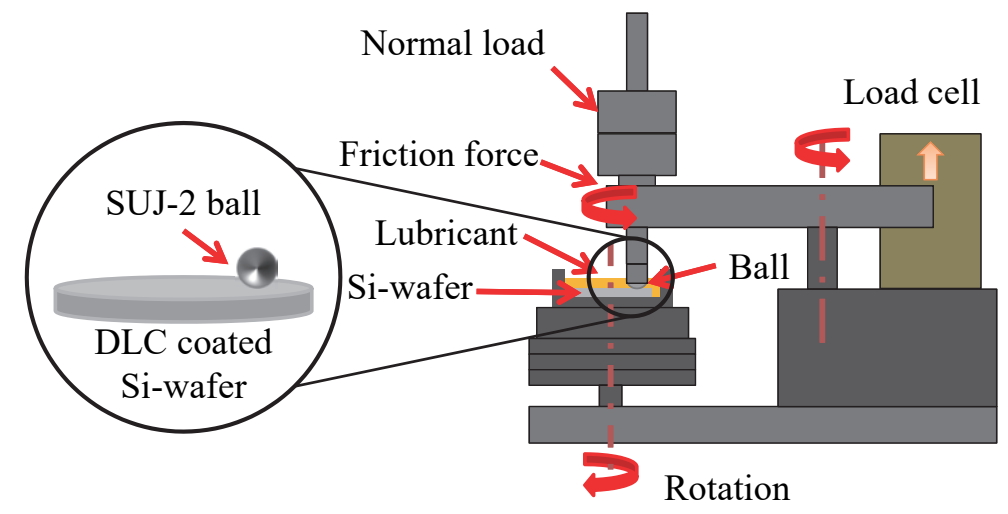

Fig. 6 Tribo-tester schematic and ball-on-disk configuration

\section{Fracture-toughness quantification for DLC coatings from radial-cracks on Si-substrate}

The indentation of the coating or bending of the substrate could produce three forms of cracking patterns namely, circumferential cracking and spallation, channel cracking and radial cracking. The aforementioned cracking modes can be applied for quantitative examination of the fracture-toughness of the coating [14].

Indentation tests were conducted via the micro Vickers hardness testing machine (Mitutoyo, Japan, 810-125 HM-102) with loads of $0.1,0.2,0.3,0.5$ and $1.0 \mathrm{kgf}$. Figure 7 illustrates that the criterion of a median crack was $c>2 a$, where $c$ was the crack length quantified starting at the center of the indentation mark to the tip and 2a was the indentation mark diagonal length [15]. Field emission scanning electron microscope FE-SEM (JEOL, JSM-7000FK) was utilised to quantify the length of the crack in which average of six indentations generated for every load was determined. K. Niihara et al. [16] demonstrated that at lower amount of crack-to-indent (c/a) ratio, palmqvist crack might form, whereas at greater amount of crack-to-indent (c/a) ratio generates median (half penny) cracks.

A previous study demonstrated the method applied for fracture-toughness determination from crack that was produced on the DLC coating/Si system [6]. The radial cracks formed on the DLC/Si system were revealed as mode 1 cracks, which the semi-circular half-penny crack geometry with full adhesion of DLC coating over the Si substrate. Hence, the fracturetoughness is denoted as;

$$
K_{f}=\sqrt{G E}
$$

Where $K_{f}$ is the fracture-toughness, $E$ is modulus of elasticity of the coating, and $G$ denotes the total work of fracture. The amount of work for elastic/brittle fracture is the amount of work to form two novel surfaces. The system fracture-toughness is measured by the combination of the total work of fracture of the system and the composite modulus of the system, which include substrate and the coating. Applying boundary condition at thickness of 0; effective fracture-toughness, $K_{r}=\sqrt{G_{s} / E_{s}}$ and $c=c_{0}$ yield;

$$
\left[\left(\frac{c_{0}}{c}\right)^{3}-1\right]=\frac{2 d}{\pi c}\left(\frac{G_{f}}{G_{s}}-\frac{E_{f}}{E_{s}}-2\right)
$$

Where $c$ and $c_{0}$ is the half radial crack length of the coated substrate and uncoated substrate, correspondingly, $E_{s}$ is the elastic modulus of the substrate, and $E_{f}$ is the elastic modulus of the film, $G_{S}$ and $G_{f}$ are the work of fracture for Si-substrate and DLC coating, respectively. The fracture of the Si-substrate is elastic and the $G_{s}$ for Si-wafer is measured by surface energy, which is $3.03 \mathrm{~J} / \mathrm{m}^{2}[17,18]$.

\section{Result and discussion}

5.1 Friction and wear

Figures 8 and 9 illustrate the coefficients that were plotted against the number of cycles for each coating and the average value, correspondingly. The average amount of friction coefficient was quantified from the coefficient of friction 
Mohd Muhyiddin Bin Mustafa, Noritsugu Umehara, Takayuki Tokoroyama, Motoyuki Murashima, Akinori Shibata, Yoshiharu Utsumi and Hideki Moriguchi

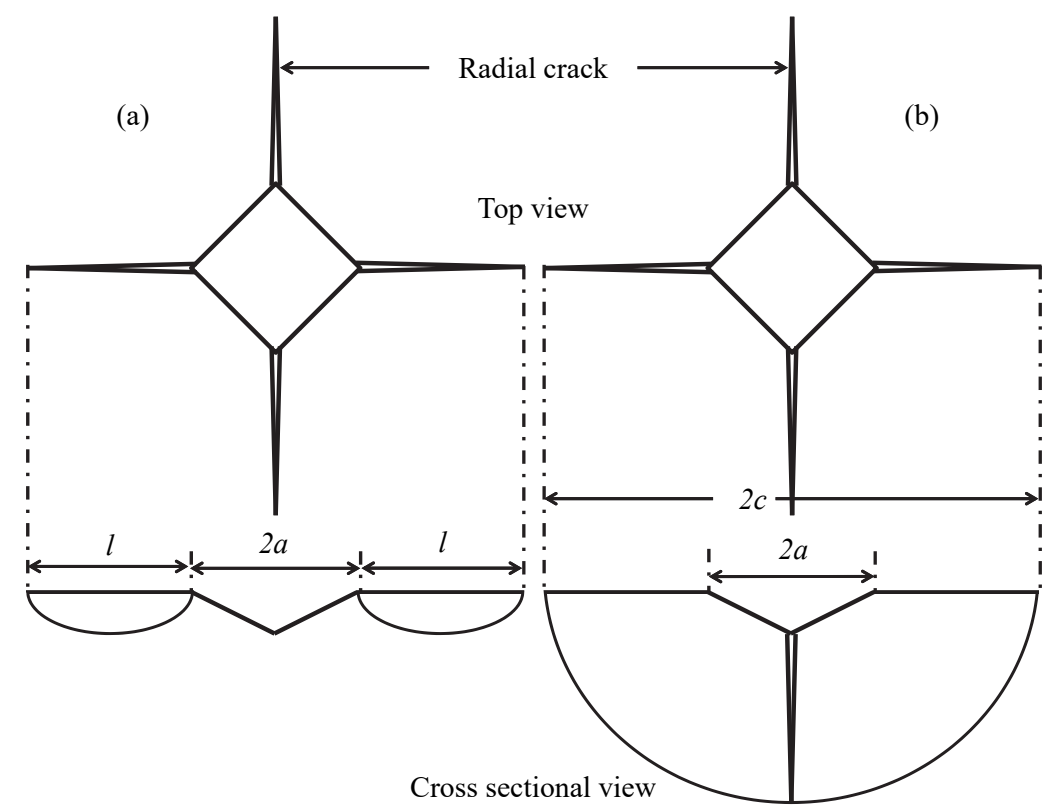

Fig. 7 Top and cross-sectional views of cracks (a) lateral (Palmqvist), and (b) median (half-penny)

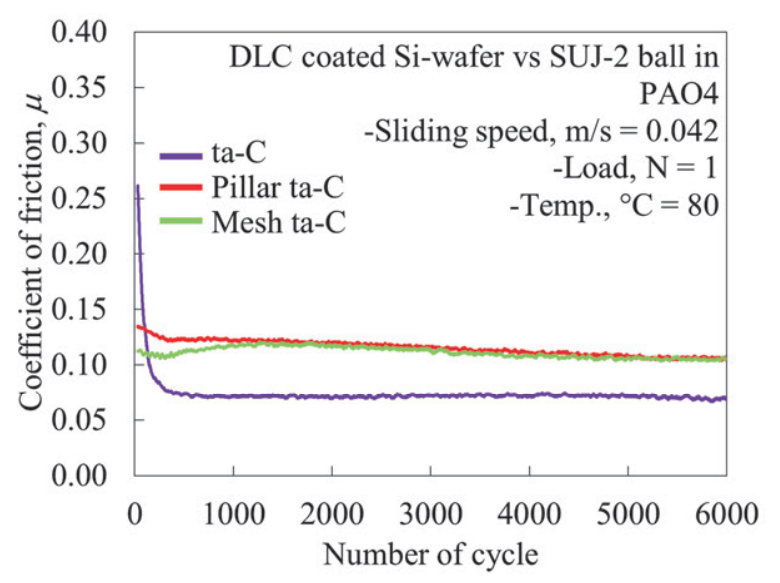

Fig. 8 Coefficient of friction result for ta-C, pillar ta-C, and mesh ta-C as a function of number of cycle

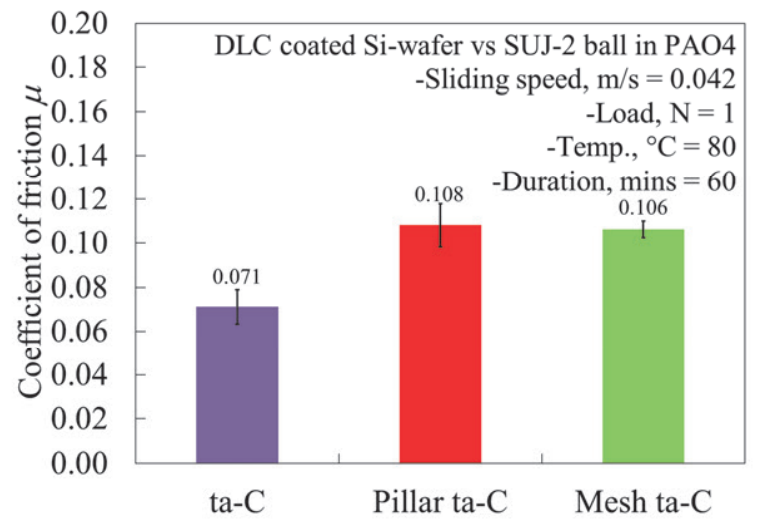

Fig. 9 Average coefficient of friction result for ta-C, pillar ta-C, and mesh ta-C under $1 \mathrm{~N}$ load

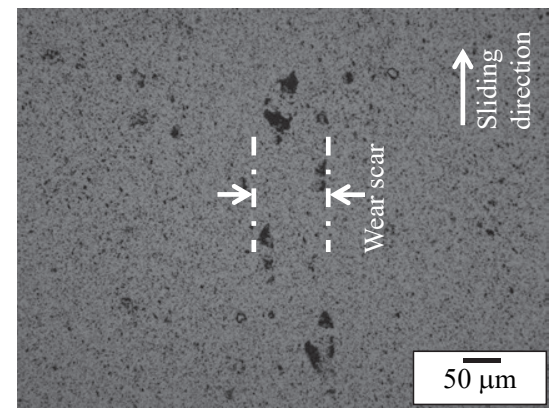

(a)

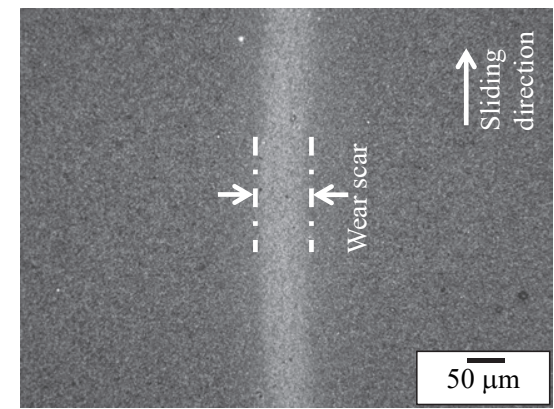

(b)

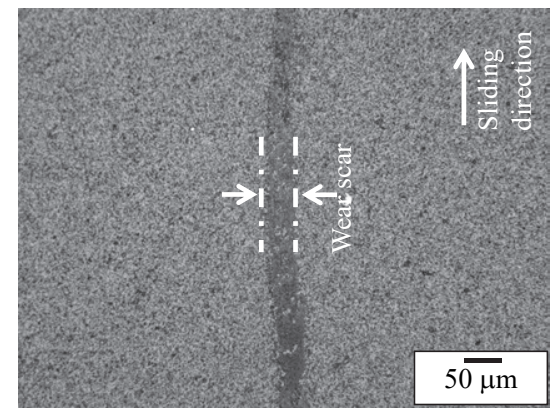

(c)

Fig. 10 Optical microscope images of the wear track on the (a) ta-C, (b) pillar ta-C, and (c) mesh ta-C under $1 \mathrm{~N}$ loads

reading at the last 2000 cycles. The result of coefficient of friction for each coating was ranged from 0.071 to 0.108 where conventional ta-C had the lowest value. In addition, the Pillar ta-C and Mesh ta-C demonstrated a higher value for coefficient of friction because of the greater average surface roughness, $R a$ as indicated by a previous study [19]. The coefficient of friction for the Pillar and Mesh ta-C decreased as the number of cycles increased. This is caused by the reduction of the surface asperities due to the deposition process, which consequently lowered the average surface roughness.

The DLC-coated Si-wafer was used to measure specific wear rates for each friction test. This was done to determine the impact of DLC structure on wear behaviour. Figure 10 illustrates the wear track generated on each coating that was detected via 

of the Coating

the optical microscope. A number of spalling areas observed on the wear track of the conventional ta-C are illustrated in Fig. 10(a). Additionally, the wear rate was determined through the 3D measuring laser microscope (Olympus, LEXT OLS5000). Figure 11 indicates the results of specific wear rates for all three types of DLC coatings under the similar applied normal loads. The application of Pillar and Mesh to the ta-C coating enhanced

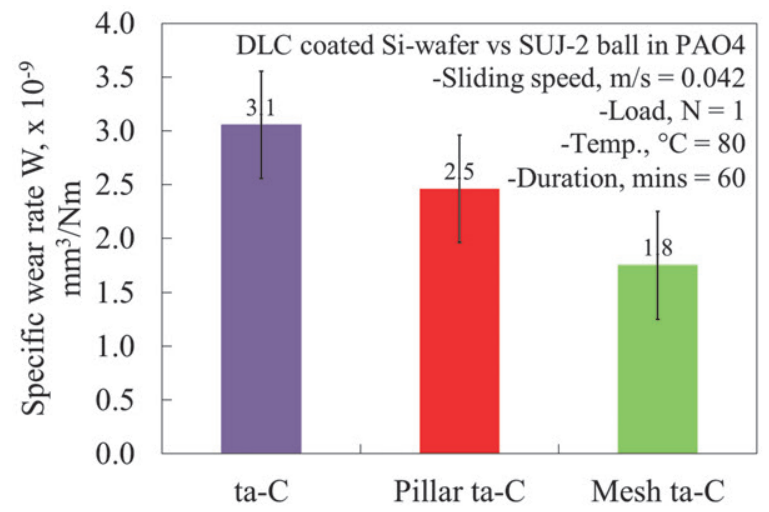

Fig. 11 Specific wear rates result for ta-C, pillar ta-C, and mesh ta- $C$ coated specimen

a(i)

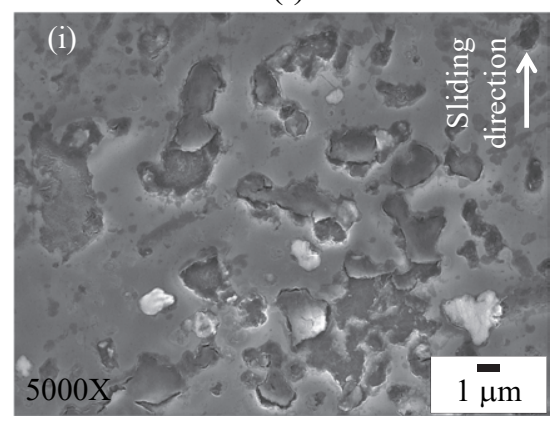

a(ii)

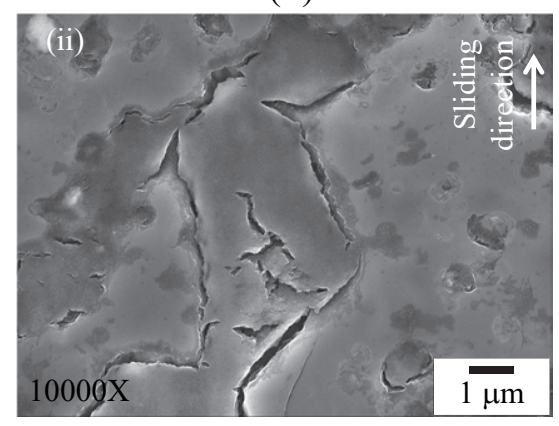

a(iii)

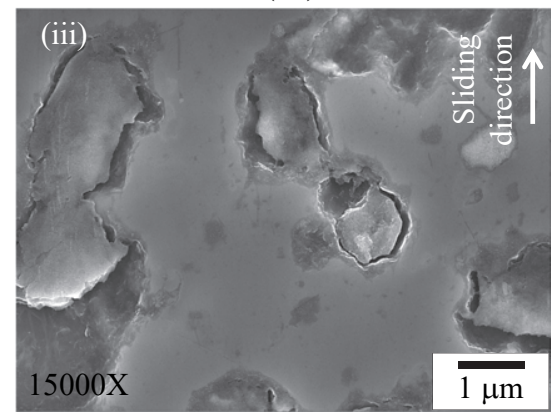

the wear resistance of the DLC coating. Mesh ta-C revealed a superior wear resistance with specific wear rates approximately $50 \%$ in comparison with conventional ta-C. The findings were in accordance with a preceding research that utilised the DLC-coated cylindrical-pin-on-disk friction test under PAO4 boundary lubrication condition [19].

A detailed analysis was conducted on the wear track of each DLC coating via the FE-SEM instrument. The wear track of the conventional ta- $\mathrm{C}$, Pillar ta- $\mathrm{C}$, and Mesh ta-C are demonstrated in Fig. 12(a), (b), and (c), correspondingly. For the case of taC, brittle form of cohesive micro-crack can be detected on the wear scar, which caused the destruction of the coating by spalling and also delamination, Fig. 12(a). Similarly, the crack was formed as the result of the deformation of the coating despite the Pillar ta-C demonstrated a few crack formations on the wear track of the coating. Evidence revealed that the crack formations were not generating any spallation of the coating. The deformation area observed at the high $\mathrm{sp}^{2}$ fraction, which consequently resulted in the deformed coating to be adhered to the local area, Fig. 12(b). Moreover, only tiny crack can be detected on the wear track of Mesh ta-C as illustrated in Fig. 12(c). Additionally, the elimination of nano-size coating from the contact area occurred in the area of $\mathrm{sp}^{2}$ rich structure was also observed. b(i)

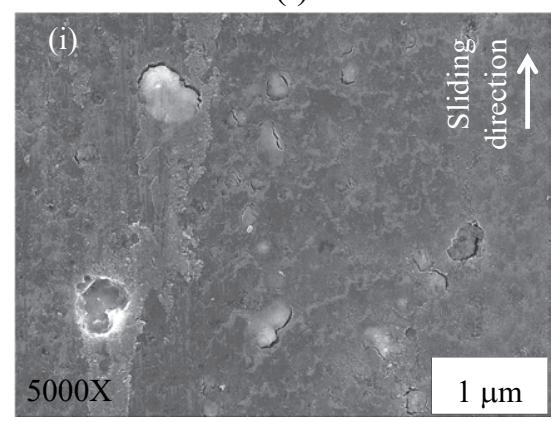

b(ii)

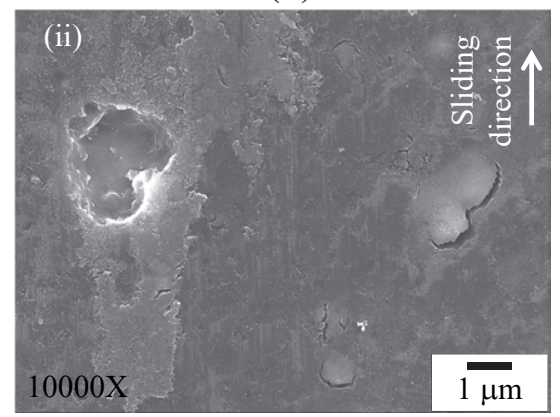

b(iii)

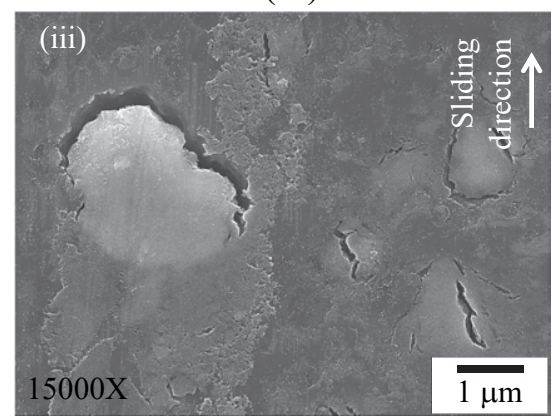

c(i)

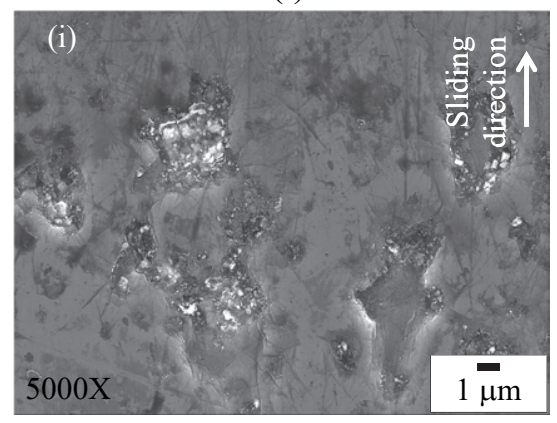

c(ii)

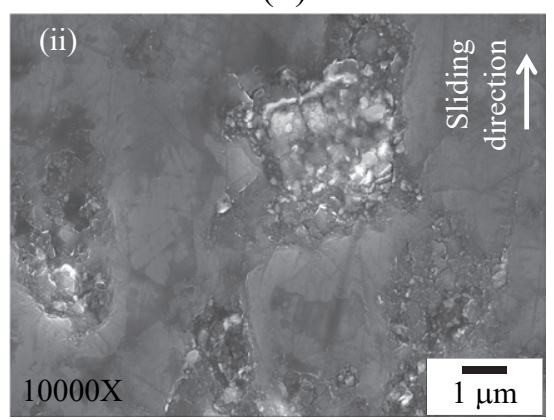

c(iii)

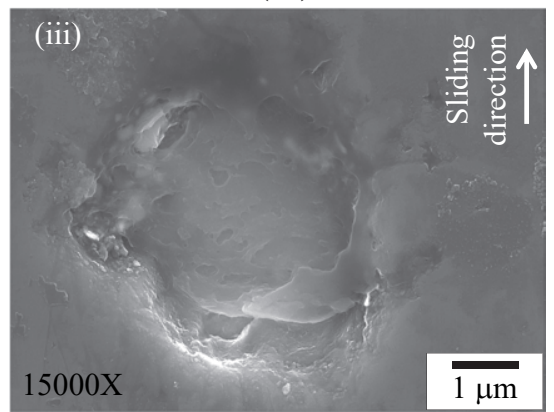

Fig. 12 FE-SEM images of the wear track on the (a) ta-C, (b) pillar ta-C, and mesh ta-C specimens under $1 \mathrm{~N}$ loads 

and Hideki Moriguchi

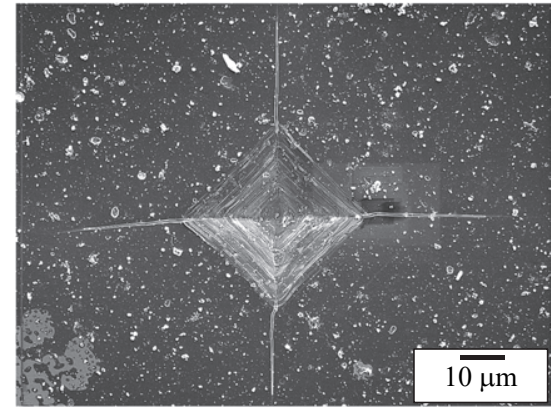

(a)

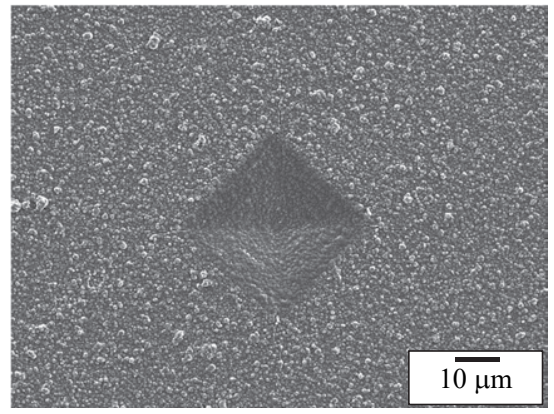

(b)

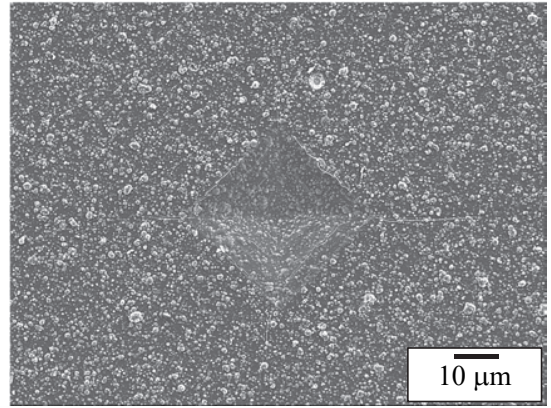

(c)

Fig. 13 Indentation spot on the (a) ta-C, (b) pillar ta-C, and (c) mesh ta-C under $10 \mathrm{~N}$ loads

There was a reduction in the number and size of the crack generated on the wear track of Pillar ta- $\mathrm{C}$ and Mesh ta- $\mathrm{C}$ coating in contrast to the conventional ta- $\mathrm{C}$ following the introduction of the Pillar and Mesh structure to the ta-C. Subsequently, the dependence of the wear to the fracture toughness of the coating was determined.

\subsection{Fracture-toughness of the coatings}

Figure 13 demonstrates the radial crack length, $c$ that was quantified on the indentation spot for every coating corresponding to the load. As the substrate work of fracture $G_{s}$ is fixed, therefore the film work of fracture $G_{f}$ can be calculated using the slope of the trend line by plotting the terms $\left[\left(c_{0} / c\right)^{3}-1\right]$ against $1 / c$ of Eq. (2) as depicted in Fig. 14 .

Table 2 depicts the findings of the fracture-toughness analysis. Mesh ta-C demonstrated the highest fracturetoughness with $16.7 \mathrm{MPa} \cdot \mathrm{m}^{1 / 2}$, followed by the Pillar ta-C with 13.4 MPa $\mathrm{m}^{1 / 2}$, and ta-C with $11.3 \mathrm{MPa} \cdot \mathrm{m}^{1 / 2}$. Introduction of structure within the ta-C increased the coatings fracturetoughness, which was linked with the greater resistance to crack. In this study, FE-SEM was utilised to detect the

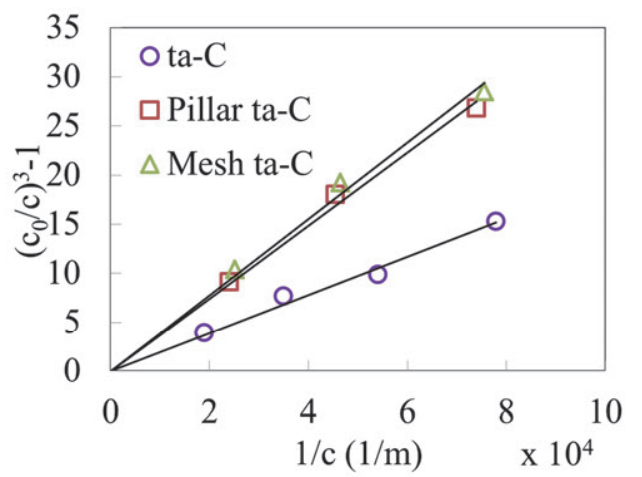

Fig. 14 The crack length data for the a-C:H, ta-C, pillar and mesh structure ta- $C$ samples plotted following the format of Eq. (2) indentation spot and the crack on conventional ta-C, Pillar and Mesh structure ta-C as shown in Figs. 15 and 16. This was done to further elucidate the involvement of the structure in improving the fracture-toughness.

The comparison of the fracture mechanism was performed by evaluating the characteristics of the crack formed on each of the coatings. Primarily, there were two forms of crack generated on DLC coating following indentation procedure. On that account, the ring crack was generated by tensile stress produced near contact area of indenter under loading process, and the spiral crack was developed during unloading process [20]. The formation of ring crack in a thin film is governed by the substrate plastic zone under the indenter [14]. This effect becomes ineffective for the thick film due to the ratio of the film thickness and the critical depth. Furthermore, during the withdrawal of the indenter, a small defect grows to spiral crack, which then extended by the arising of equi-biaxial stress field due to film bending curvature effect [20,21].

The investigation within the indentation mark for Pillar and Mesh ta-C coating demonstrated that every crack produced was detached and revealed a zigzag path pattern, which was corresponded to a mixture of Modes I and II cracks [22], that consequently resulted in ductile cracking as illustrated in Fig. 15(b), and (c), correspondingly. This zigzag or crinkled crack pattern provided stress relieve by permitting greater rate of crack energy dissipation in comparison with ta-C coating. In addition, the ta-C also demonstrated smooth step/layer crack shape and transverse crack in the indentation mark as depicted in Fig. 15(a). This step/layer crack pattern was elucidated by the brittleness of the ta- $\mathrm{C}$ that restrained the coating from deformation when load was induced. Additionally, Fig. 15 illustrates that spallation can only be detected in conventional ta-C. It should be noted that both Pillar and Mesh ta-C coating revealed larger cohesive cracks spacing in comparison with the conventional ta-C. The crack spacing could decline under good adhesion conditions as the results of the transfer of the misfit stress amongst the film and the substrate to the film deprived of interface failures [23-25]. As such, this revealed that the

Table 2 Result of the fracture-toughness of the coatings

\begin{tabular}{lrrrrr}
\hline Samples & $\begin{array}{c}\text { Film } \\
\text { thickness } \\
\boldsymbol{d}, \boldsymbol{\mu m}\end{array}$ & $\begin{array}{c}\text { Elastic } \\
\text { modulus } \\
\boldsymbol{E}_{\boldsymbol{f}}, \mathbf{G P a}\end{array}$ & $\begin{array}{c}\text { Compressive } \\
\text { residual stress } \\
\boldsymbol{\sigma}_{\boldsymbol{c}}, \mathrm{GPa}\end{array}$ & $\begin{array}{c}\text { Film work of } \\
\text { fracture } \\
\boldsymbol{G}_{\boldsymbol{f}}, \mathbf{k J} / \mathbf{m}^{\mathbf{2}}\end{array}$ & $\begin{array}{c}\text { Fracture-toughness } \\
\boldsymbol{K}_{\boldsymbol{f}}, \mathbf{M P a} \cdot \mathbf{m}^{\mathbf{1 / 2}}\end{array}$ \\
\hline ta-C & 1.5 & 310 & 1.75 & 0.45 & 11.3 \\
Pillar ta-C & 1.42 & 143 & 1.26 & 1.25 & 13.4 \\
Mesh ta-C & 1.25 & 184 & 2.15 & 1.14 & 16.7 \\
\hline
\end{tabular}



of the Coating

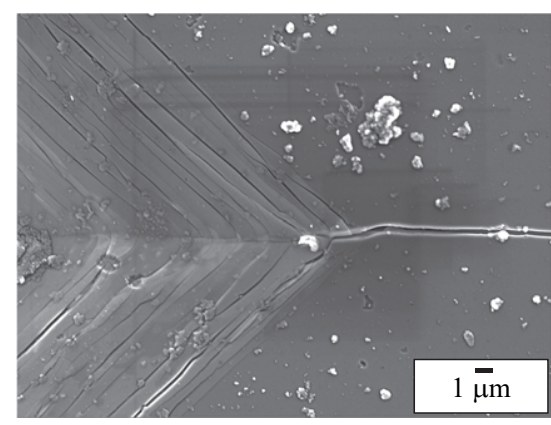

(a)

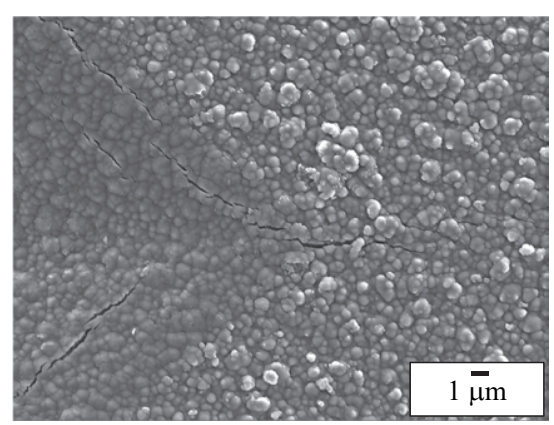

(b)

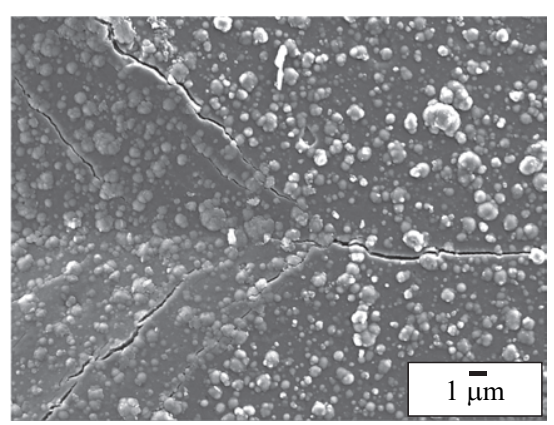

(c)

Fig. 15 Magnified indentation spot on the (a) ta-C, (b) pillar ta-C, and (c) mesh ta- $\mathrm{C}$ under $10 \mathrm{~N}$ loads

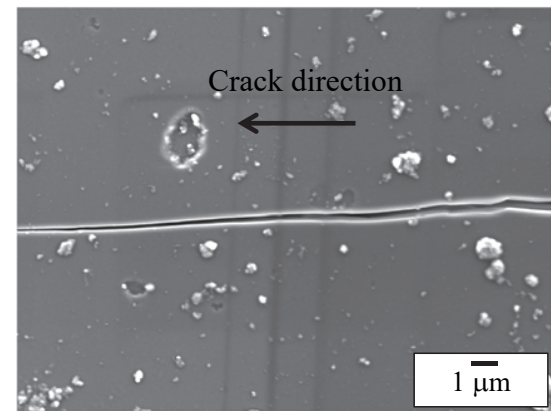

(a)

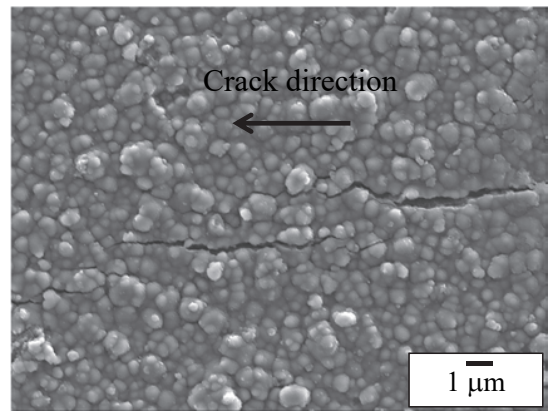

(b)

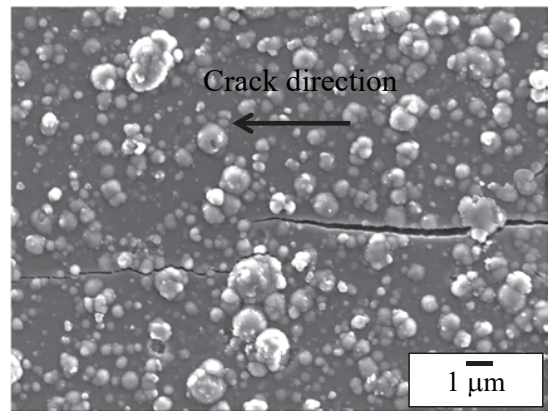

(c)

Fig. 16 Magnified FE-SEM images of the radial cracks on the (a) ta-C, (b) pillar ta-C, and (c) mesh ta-C under $10 \mathrm{~N}$ loads

adhesion strength was elevated by the introduction of the Pillar and Mesh structure to the ta-C.

Notably, the ta-C indentation mark diagonal length (2a) was comparable to that of Pillar and Mesh type ta-C although ta-C DLC coating hardness is approximately two times higher than that of structured ta-C as depicted in Fig. 13. As such, this is the impact of greater elastic recovery value, $W_{e}$ of the Pillar and Mesh ta-C in comparison with homogeneous ta-C DLC coating as demonstrated in Fig. 17. As such, this could result in greater degree of coating toughness $[13,26] . W_{e}$ can be obtained from the typical load-displacement curves as summarized in Eq. (3).

$W_{e}=\left[\left(d_{\max }-d_{r e s}\right) / d_{\max }\right] \times 100 \%$

Where;

- $d_{\max }=$ maximum indentation depth during loading

$-d_{r e s}=$ residual indentation depth after unloading

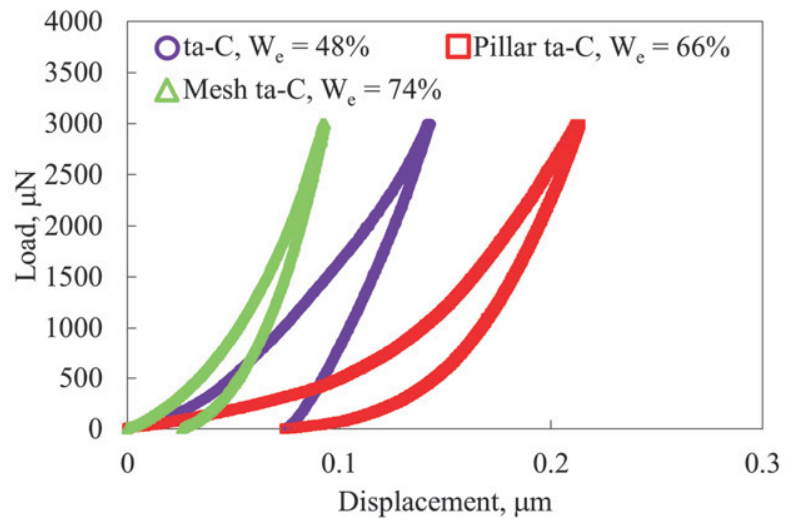

Fig. 17 Elastic recovery value, $W_{e}$ for DLC coatings from loading-unloading curves during indentation test
Figure 16 demonstrates the radial channel-type cohesive cracking on every form of DLC coating. The cohesive crack has a vital function as a flaw starting the interface delamination [27]. Following subsequent straining, further buckling delamination happened at the free edge of the cohesive crack of ta-C coating because of the increased energy release rate and compressive strain that was generated by Poisson's contraction normal to the tensile direction as illustrated in Figs. 15(a) and 16(a) [28]. Finer radial crack can be detected on the ta-C coating, which is in comparison with the radial crack of Pillar and Mesh ta-C as depicted in Fig. 16(a), (b), and (c), correspondingly. Inferior fracture-toughness of the Pillar ta-C to that of Mesh ta-C was caused by its lower hardness features and elastic recovery value (Fig. 17). Figure 16(b) depicts the features of the pillar structure that facilitated the formation of longer parallel crack. Mesh ta- $\mathrm{C}$ and Pillar ta-C demonstrated a shorter crack length in comparison with that of ta-C coating, indicating conventional ta-C had lower fracture-toughness. On the other hand, higher coating fracture-toughness restrained the crack propagation, consequently inhibited coating from spalling and delamination. The combined impact of soft $\mathrm{sp}^{2}$ structure and intersection restricted the crack from propagating in Pillar ta-C and Mesh taC. Higher rate of energy dissipation via plastic deformation of the $\mathrm{sp}^{2}$ phase soft structure resulted in an improvement in film toughness, which subsequently caused the termination of the crack propagation [29].

\section{Fracture-toughness in relations to wear rates}

The wear rates for non-structured and structured ta-C were plotted against fracture-toughness of the coating to examine the link between the crack resistance to the wear rates of the coating (Fig. 18). The characterisation of the coating by virtue of its mechanical properties is crucial to provide detailed information 


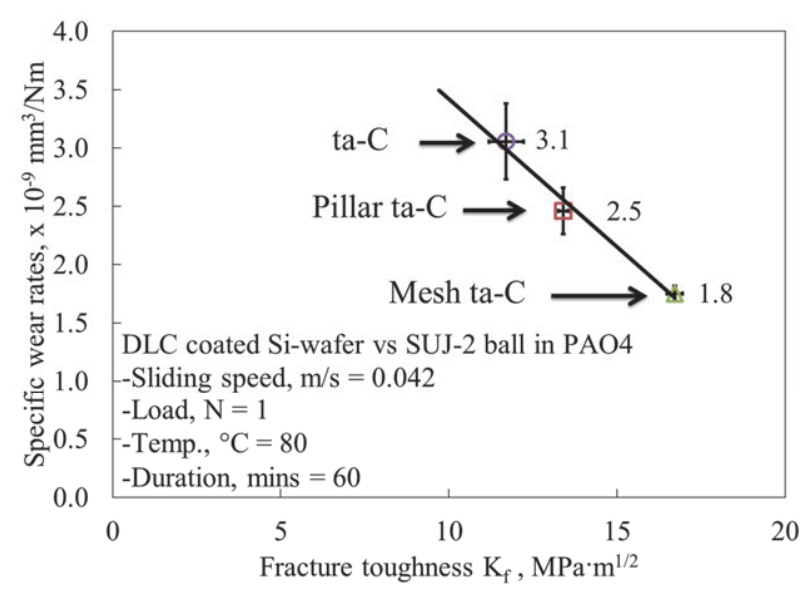

Fig. 18 Specific wear rates plotted against fracture-toughness of the coating for DLC-coated Si-disk vs SUJ-2 ball

on the tribological procedure in the boundary lubrication regime as direct contact of surface asperities exist. There was an inverse relationship between the wear rates and the fracturetoughness, at which the wear rates were declined following the improvement in the fracture-toughness. The findings revealed that the corresponding wear resistance was increased following the improvement in the fracture-toughness of the coating. As such, higher Pillar and Mesh ta-C coating resistance to wear was observed as the results of an elevated fracture-toughness. This consequently declined the coating destruction as an effect of fracture-induced wear in conventional ta-C that further evolved to spalling and delamination of the coating. The small fragments spall ta-C coating could then act as an abrasive particle to promote the wear acceleration.

\section{Conclusion}

In order to discuss about the effect of structure of Pillar and Mesh to the ta-C wear properties, fracture-toughness evaluation via micro indentation for these various ta-C was evaluated. In addition, the effect of fracture-toughness of the DLC on wear properties in oil lubrication was clarified. Superior fracturetoughness was observed following the introduction of pillar and mesh structure to the ta- $\mathrm{C}$ coating in which the fracturetoughness of the Pillar and Mesh ta-C DLC-coatings were comparatively higher than the conventional ta-C. Furthermore, elevation of fracture-toughness led to higher wear resistance of the ta-C coating due to the fracture-induced wear suppression through improvement of the crack propagation inhibition. In addition, introduction of Pillar and Mesh structure to the ta-C DLC coating prevented the brittle characteristics of the ta-C by decreasing channel-type cohesive cracking. Moreover, Pillar and Mesh structure ta- $C$ provided greater degree of stress relieve by permitting higher rate of crack energy dissipation in contrast to conventional ta-C coating. Also, the increased crack spacing in Pillar and Mesh ta-C was facilitated by an improved adhesion strength, which prevented the interface failures. The friction coefficient for Pillar ta-C and Mesh ta-C are comparatively higher to that of ta- $C$ due to higher contact surface roughness.

\section{Acknowledgement}

The author, Mohd Muhyiddin Bin Mustafa would like to thank the Ministry of Education, Malaysia as well as Universiti
Teknikal Malaysia Melaka (UTeM) for the financial support provided for pursuing his doctoral study.

\section{References}

[1] Rasel, S. M., Wang, Y. Q., Ku, H. K., Byeon, J. M., Kim, T. K. and Song, J. I., "Determination of Fracture Toughness of Amorphous Carbon Coatings Using Indentation Method," Proc. International Conference on Composite Materials, Korea, 2011, 5-8.

[2] Wang, Y. and Yu, X., "Exploring Film Failure Mechanism and Scratch Toughness Characterization of Diamond-Like Carbon Film," Proc. International Conference on Chemical, Material and Food Engineering, China, 2015, 306-309.

[3] Bull, S. J., "Tribology of Carbon Coatings: DLC, Diamond and Beyond," Diamond Related Materials, 4, 5-6, 1995, 827-836.

[4] Li, X., Deng, X., Kousaka, H. and Umehara, N., “Comparative Study on Effects of Load and Sliding Distance on Amorphous Hydrogenated Carbon (a-C:H) Coating and Tetrahedral Amorphous Carbon (ta-C) Coating under Base-Oil Lubrication Condition," Wear, 392-393, 2017, 84-92.

[5] Van der Donck, T., Muchlado, M., Zein Eddine, W., Achanta, S., Carvalho, N. J. M. and Celis, J. P, “Effect of Hydrogen Content in a-C:H Coatings on Their Tribological Behaviour at Room Temperature up to $150^{\circ} \mathrm{C}$," Surface and Coatings Technology, 203, 22, 2009, 3472-3479.

[6] Nastasi, M., Kodali, P., Walter, K. C. and Embury, J. D., "Fracture Toughness of Diamond-Like Carbon Films," Journal of Materials Research, 15, 2001, 157-162.

[7] Leung, D. K., He, M. Y. and Evans, A. G., "The Cracking Resistance of Nanoscale Layers and Films," Journal of Materials Research, 10, 7, 1995, 1693-1699.

[8] Feng, Y., Zhang, T. and Yang, R., "A Work Approach to Determine Vickers Indentation Fracture Toughness," Journal of the American Ceramic Society, 94, 2, 2011, 332-335.

[9] Chicot, D., Pertuz, A., Roudet, F., Staia, M. H. and Lesage, J., "New Developments for Fracture Toughness Determination by Vickers Indentation," Materials Science and Technology, 20, 7, 2004, 877884.

[10] Kalpakjian, S., "Manufacturing Engineering and Technology," Pearson/Prentice Hall, 2005, 1295.

[11] Kruzic, J. J. and Ritchie, R. O., "Determining the Toughness of Ceramics from Vickers Indentations Using the Crack-Opening Displacements: An Experimental Study," Journal of the American Ceramic Society, 86, 8, 2003, 1433-1436.

[12] Sui, X., Liu, J., Zhang, S., Yang, J. and Hao, J., “Microstructure, Mechanical and Tribological Characterization of CrN/DLC/Cr-DLC Multilayer Coating with Improved Adhesive Wear Resistance," Applied Surface Science, 439, 2018, 24-32.

[13] Guo, C. Q., Pei, Z. L., Fan, D., Gong, J. and Sun, C., “Microstructure and Tribomechanical Properties of $(\mathrm{Cr}, \mathrm{N})$-DLC/DLC Multilayer Films Deposited by a Combination of Filtered and Direct Cathodic Vacuum Arcs," Diamond and Related Materials, 60, 2015, 66-74.

[14] Zhang, S. and Zhang, X., "Toughness Evaluation of Hard Coatings and Thin Films," Thin Solid Films, 520, 7, 2012, 2375-2389.

[15] Antis, G. R., Chantikul, P., Lawn, B. R. and Marshall, D. B., “A Critical Evaluation of Indentation Techniques for Measuring Fracture Toughness: I, Direct Crack Measurements," Journal of the American Ceramic Society, 64, 9, 1981, 533-538.

[16] Niihara, K., Morena, R. and Hasselman, D. P. H., “Evaluation of $K_{\mathrm{lc}}$ of Brittle Solids by the Indentation Method with Low Crack-toIndent Ratios," Journal of Materials Science Letters, 1, 1, 1982, 13-16.

[17] Jaccodine, R. J., "Surface Energy of Germanium and Silicon," Journal of the Electrochemical Society, 110, 6, 1963, 524-527. 

of the Coating

[18] Gilman, J. J., "Direct Measurements of the Surface Energies of Crystals," Journal of Applied Physics, 31, 12, 1960, 2208-2218.

[19] Mustafa, M. M. B., Umehara, N., Tokoroyama, T., Murashima, M., Shibata, A., Utsumi, Y. and Moriguchi, H., "Effect of Mesh Structure of Tetrahedral Amorphous Carbon (ta-C) Coating on Friction and Wear Properties under Base-Oil Lubrication Condition," Tribology International, 2019.

[20] Yonezu, A., Nakayama, T. and Ogawa, T., "Fracture Mechanism of Diamond Like Carbon (DLC) Film Subjected to Contact Loading," Journal of the Society of Materials Science, Japan, 57, 5, 2008, 474480 (in Japanese).

[21] Fu, K., Chang, L., Ye, L. and Yin, Y., "Indentation Stress-Based Models to Predict Fracture Properties of Brittle Thin Film on a Ductile Substrate," Surface and Coatings Technology, 296, 2016, 4657.

[22] Li, M., Deng, T., Zheng, B., Zhang, Y., Liao, Y. and Zhou, H., “Effect of Defects on the Mechanical and Thermal Properties of Graphene," Nanomaterials, 9, 3, 2019, 347.

[23] Latella, B. A., Triani, G. and Evans, P. J., "Toughness and Adhesion of Atomic Layer Deposited Alumina Films on Polycarbonate
Substrates," Scripta Materialia, 56, 6, 2007, 493-496.

[24] Jeong, J. H. and Kwon, D., "Evaluation of the Adhesion Strength in DLC Film-Coated Systems Using the Film-Cracking Technique," Journal of Adhesion Science and Technology, 12, 1, 1998, 29-46.

[25] Kim, H. J., Moon, M. W., Kim, D. I., Lee, K. R. and Oh, K. H., "Observation of the Failure Mechanism for Diamond-Like Carbon Film on Stainless Steel under Tensile Loading," Scripta Materialia, 57, 11, 2007, 1016-1019.

[26] Rupa, P. K. P., Chakraborti, P. C. and Mishra, S. K., "Structure and Indentation Behavior of Nanocomposite Ti-B-N Films," Thin Solid Films, 564, 2014, 160-169.

[27] Moon, M. W., Chung, J. W., Lee, K. R., Oh, K. H., Wang, R. and Evans, A. G., "An Experimental Study of the Influence of Imperfections on the Buckling of Compressed Thin Films," Acta Materialia, 50, 5, 2002, 1219-1227.

[28] Hutchinson, J. W. and Suo, Z., "Mixed Mode Cracking in Layered Materials," Advances in Applied Mechanics, 29, 1991, 63-191.

[29] Subramanian, C. and Strafford, K. N., "Review of Multicomponent and Multilayer Coatings for Tribological Applications," Wear, 165, 1 , 1993, 85-95. 